\title{
Implementation of Two-Axis Position-Based Impedance Control with Inverse Kinematics Solution for A 2-DOF Robotic Finger
}

\author{
Khairunnisa Nasir, Ruhizan Liza Ahmad Shauri*, Norshariza Mohd Salleh, Nurul Hanani Remeli, \\ Faculty of Electrical Engineering, Universiti Teknologi MARA, 40450 Shah Alam, Selangor, Malaysia \\ *Corresponding author E-mail: ruhizan@salam.uitm.edu.my
}

\begin{abstract}
Position-based impedance control is a force control approach which consists of a single control law that accommodates the external force to achieve the desired dynamics of the body. A previously developed three-fingered robot hand was very rigid in its motion due to the application of position control alone. The position control scheme was inadequate for the tasks that involves the interaction of a robot end-effector with its environment which could damage fragile objects or be prone to slippage when provided with incorrect object's position. This paper introduces the application of two-axis position-based impedance control to one of the 2 degree-of-freedom (DOF) robotic finger of the robot hand. The goal of the control is to produce a mass-spring-dashpot system for the robot hand which considers the external force exerted by the object or environment onto the finger to modify the targeted position of the robot's tip-end. The positionbased impedance control which was successfully performed however could not directly drive the DC-micromotors at the finger joints since it was expressed in the Cartesian position $(\mathrm{X}, \mathrm{Y}, \mathrm{Z})$ form. Therefore, inverse kinematics was derived using geometrical approach to convert the Cartesian position $(\mathrm{X}, \mathrm{Y}, \mathrm{Z})$ to angle position of motor which is controlled by PID. The proposed control and the developed kinematics were programmed using Matlab Simulink and tested in real-time experiments. The validation result has proven that the proposed position-based impedance control could modify the initial fingertip position according to the amount and direction of the applied external force, thus produced softness to the robotic finger.
\end{abstract}

Keywords: End-effector; force control; impedance control; inverse kinematics; robot finger; robot hand.

\section{Introduction}

Force control is very important for many robot manipulators as their practical manipulation tasks are normally associates with interaction between the end-effector of the robot and the environment. In current trend, robot manipulators are supposed to be more autonomous in which a simple motion control is inadequate to execute variety of manufacturing tasks where object sizes and positions may vary.

Various force control strategies have been developed for different research applications to satisfy the requirement of tasks performed by robots. According to Patel et al. [1], position control schemes are inadequate for the tasks that involves the interaction of a manipulator with its environment. Force control schemes are required to be developed in order to cater the task that demands extensive contact with the environment. Garcia et al. [2] claimed that in robotics manipulation task, manipulation can be performed only after the interaction forces are controlled properly, thus force control is required.

Impedance control is a well established force control scheme which was first introduced by Hogan [3] to establish a dynamical relationship between the force and robot end-effector position by regulating the mechanical impedance $[4,5]$. According to Siciliano and Villani [6], to achieve a desired dynamic behavior, the impedance parameters known as mass, damping and stiffness need to be considered at the contact. An appropriate force/torque sensor usually mounted at the manipulator wrist is used to acquire information on the contact force and moment to keep linearity and decoupling of the system during the interaction with the environment. Furthermore, the environmental forces and torques can be measured by a $6-\mathrm{DOF}$ force-torque sensor mounted at the tip of the robot in usual industrial scenarios [7].

In principle, the end-effector can be controlled either as an actuator of force and torque or an actuator of position depending on the causality of the controller [8]. Controller with impedance causality is termed as force/torque-based impedance control while controller with causality of a mechanical admittance is termed as position-based impedance control $[5,8,9]$. In the force/torque-based impedance control, the end-effector is controlled by treating the mechanism as an actuator of force and torque to satisfy the impedance objective. The positional deviations are measured explicitly through position sensor while force and torque are commanded to actuate the end-effector $[8,10]$. In contrast, the position-based impedance control controls the end-effector by treating the mechanism as an actuator of position. The position-based impedance control is a very well approach that suits for industrial robots as these robots are generally equipped with position controllers.

The previously developed robot hand was very rigid in its motion due to the application of position control alone [11]. Furthermore, the application of position control alone could damage fragile objects or be prone to slippage when provided with an incorrect object's position. This situation happens due to the absence of touch sensing ability of the robotic finger when interacting with the environment or an object. Therefore, this study introduces the position-based impedance control in two individual directions namely $x$-axis and $z$-axis focusing on one of the 2-DOF robotic finger only. The method aims to control the dynamic relation of the robot with the environment by regulating the force with ad- 
justments made to the end-effector position to react the contact forces, which producing a mass-spring-dashpot system to the 2DOF robotic finger. Prior to the position-based impedance control development, a new fingertip that embeds force sensors in $x$-axis and $z$-axis directions was designed in a separate study by Jaafar $e t$ al. [12] and Jaafar et al. [13]. Furthermore, calibration work for force measurement with the new design fingertip was successfully done in [14]. The position-based impedance control is implemented with PID position control for driving the motors through the inverse kinematics derivation. The inverse kinematics is derived to convert the modified position resulted from the position-based impedance control to angle references for actuation of DCmicromotors located at each joint of the robotic finger. The algorithms were developed in Matlab Simulink and validated through real-time experiments. Finally, the experimental results are analyzed and the discussions on the output system performance are presented.

\section{Robot Hand Structure and System}

The robot hand consists of a 1-DOF palm and three 2-DOF fingers which make the total of 7-DOF for the whole hand. One finger is fixed at the palm while the other two fingers are movable synchronously along the rotation of z-axis direction produced by the joint at the palm. Each finger has two joints actuated by

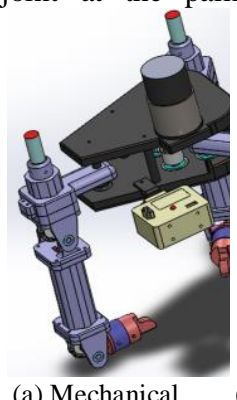

(a) Mechanical design robot hand

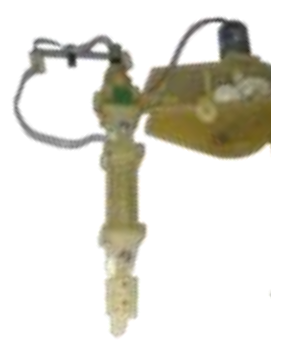
hand. (b) Fabricated robot

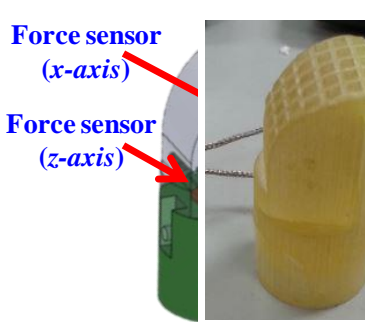

(c) Mechanical design robotic finger. (d). Fabricated robotic finger.
Fig. 1: Three-fingered robot hand [11] [13].

\section{Position-Based Impedance Control}

The position-based impedance control was developed by considering the translational impedance equation to enforce an equivalent mass-spring-dashpot behavior for the position displacement when the end-effector exerts a force on the environment as follows;

$P_{c}=P_{d}+\Delta P$

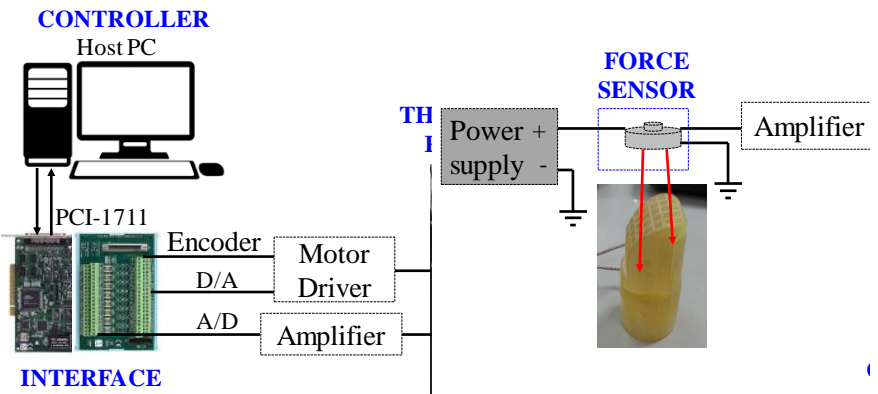
INTERFACE

Fig. 2: System architecture of the three-fingered robot hand.

FAULHABER DC-micro motor while the palm is actuated by a CYTRON DC-geared motor Fig. 1(a) depicts the complete structure of the three-fingered robotic hand while Fig. 1(b) represents the complete fabricated and assembled robotic hand. Fig. 1(c) shows the new design fingertip used in this study which two Futek LLB130 sub-miniature load cells were installed to measure the force. The fingertip was designed in separate study by Jaafar $e t$ $a l .[13]$ and fabricated using perspective plastics material as shown in Fig. 1(d).

Fig. 2 describes the communication between the controllers, interface, actuators, sensors and the robotic hand. A desktop computer was used as a host which contains the control algorithms of the robotic hand developed using Matlab Simulink. Meanwhile, a Peripheral Component Interconnect (PCI) installed in the host computer was used as an interface of the robotic hand system. The sensors and actuators were connected to the terminal board PCLD8710 that matched the PCI. The system was conducted in realtime, thus the algorithms were executed using sampling time set at $1 \mathrm{~ms}$. As shown in Fig. 3, the signal force sensed by the force sensor is amplified before sent to host PC to be controlled.

Fig. 3: System architecture of the three-fingered robot hand Force sensor circuitry. ence between the measured external force, $F_{\text {ext }}$ and a fixed force reference, $F_{r e f}$. Rearranging equation (2) can be used to calculate $P_{c}$ as shown in equation (3).

$\Delta P=P_{c}-P_{d}$

$F_{\text {ext }}-F_{\text {ref }}=M_{d}(\Delta \ddot{P})+D_{d}(\Delta \dot{P})+K_{d}(\Delta P)$

where the contact force for the robotic fingertip is given by the difference between the measured external force, $F_{\text {ext }}$ and a fixed force

reference, $F_{r e f}$. Meanwhile, $M_{d}, D_{d}, K_{d}$ are the impedance parameters known as mass, damping and stiffness coefficients which were set at value of 1,10 and 1000 , respectively. $\Delta \ddot{P}, \Delta \dot{P}$, and $\Delta P$ represent the acceleration, velocity and position of the robotic fingertip, respectively.

A reference frame known as compliant frame $\Sigma_{c}$ is introduced other than the desired frame $\Sigma_{\mathrm{d}}$ to describe the trajectory perturbation that takes the difference between the modified tip-end position $P_{c}$ and desired tip-end positions $P_{d}$ as demonstrated in equation (2). Equation (1) is used to compute $\Delta P$ and its associated derivatives with force information provided by the force sensors. The contact force for the robotic fingertip is taken from the differ-

$F_{r e f}$ is used as a threshold to activate the position-based impedance control in which the desired position $P_{d}$ will be modified to $P_{c}$ when $F_{\text {ext }}$ exceeded $F_{\text {ref }}$. When there is no interaction forces occurred on the robot's tip-end, there will be no trajectory perturbation, thus, $P_{c}=P_{d}$. Note that at this point, the position of the tipend is resulted in the form of Cartesian position $(\mathrm{X}, \mathrm{Y}, \mathrm{Z})$.

\section{Inverse Kinematics}

Inverse kinematics specifies the location of the robot's tip-end from the Cartesian position form by computing the associated joint angles of the robotic finger. It converts Cartesian position of tip-end $(\mathrm{X}, \mathrm{Y}, \mathrm{Z})$ to joint angles for the fingers to actuate the motors at each joint of the robotic finger. Geometrical approach is used to solve the inverse kinematics for one of the 2-DOF robotic finger due to its capability of finding optimal solution with less computation. The diagram of the 2-DOF robotic finger is depicted in Fig. 4(a) which the condition of the tip-end position must be in 
the

ranges

$-9.03 \mathrm{~cm}<\mathrm{X}<7.1 \mathrm{~cm} ; \mathrm{Y}=0 \mathrm{~cm}$ and $2.715 \mathrm{~cm}<\mathrm{Z}<18.86 \mathrm{~cm}$ to ensure that the robot's tip-end is within its reachability range. The point $O$ is the origin of the robot hand while point $R$ is the tipend of the robotic finger. $L_{1}, L_{2}, L_{3}$, and $L_{4}$ are the length dimensions from point $\mathrm{O}$ to $\mathrm{R}$. Meanwhile, Fig. 4(b) presents the angles that contributed to the computation of $\theta_{1}$ and $\theta_{2}$ using geometrical approach by Saiki [15]. The calculations of $\theta_{1}$ and $\theta_{2}$ are shown in equations (4), and (5) which apply fixed angle $\phi$ and all the joint variables $\alpha, \beta$, and $\gamma$ as depicted in Fig. 4(a).

$$
\begin{aligned}
& \theta_{1}=180^{\circ}-(\alpha+\beta+\phi) \\
& \theta_{2}=180^{\circ}-\gamma
\end{aligned}
$$

$\theta_{1}$ is computed using equation (4) which involves the calculation of $\alpha, \beta$ and $\phi$ as in Fig. 4(b). $\phi$ is the fixed angle which was set at $90 \operatorname{deg}\left({ }^{\circ}\right)$. Meanwhile, the joint variable $\alpha$ can be calculated using $d_{2}$ and $d_{3}$ through trigonometry and $\beta$ can be obtained by cosine rule that involves calculation of $L_{1}, L_{2}$ and $d_{3} . L_{1}$, and $L_{2}$ are known values however the value of $d_{3}$ depends on the $d_{1}$ and $d_{2}$. The values of $d_{1}, d_{2}$ and $d_{3}$ are regulated based on the position vector of $R$ related to joint 1 with respect to $z$-axis and $x$-y axis. Therefore, $d_{3}$ can be determined using Pythagoras theorem. Finally, $\theta_{2}$ is computed using equation (5) which involves $\gamma$ depicted in Fig. 4(b). The cosine rule was also implemented to obtain $\gamma$.

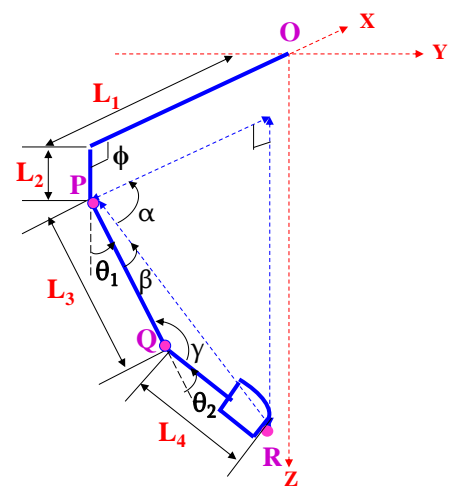

(a) Diagram of the 2-DOF robot finger.

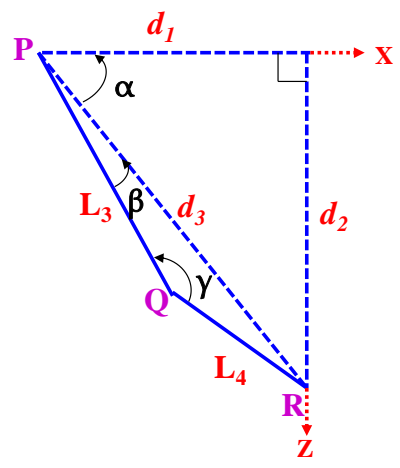

(b) Geometrical approach.

Fig. 4: Inverse kinematics solution.

\section{Work Validation}

The proposed method is validated by implementing the positionbased impedance control together with position control to ensure that the commanded trajectory could perform successfully in the real-time experiment. The results are discussed in three phases including the validation of the proposed position based-impedance control, the inverse kinematics, and the combination of both with PID control. In this work, the parameters of the PID controller were set at $K_{p}=0.8, K_{i}=1.45$ and $K_{d}=0.1$. All work phases are integrated sequentially with one and another to create the complete robotic system as presented in Fig. 5 .

$P_{d}$ was provided in Cartesian form at $[\mathrm{X}=0.04246 ; \mathrm{Y}=0$; $\mathrm{Z}=0.1245]$. Meanwhile, $F_{\text {ref }}$ was specified at $1 \mathrm{~N}$ for $x$-axis and $2 \mathrm{~N}$ for $z$-axis to activate the developed impedance control in respective axis. When the impedance control output is activated, the $P_{d}$ will be modified to $P_{c}$. Five random forces were applied and released to the robot's tip-end in the direction of $x$-axis and $z$-axis during the experiment using human finger. The output results from each integration phase were observed and analyzed to validate the proposed two-axis position-based impedance control in this study.

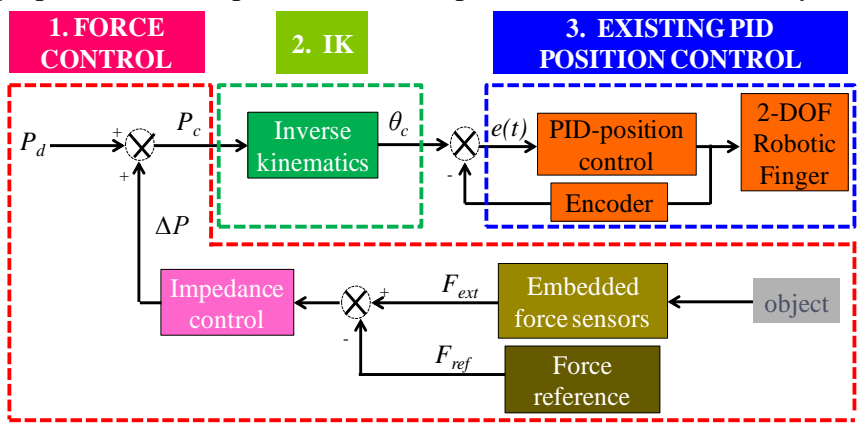

Fig. 5: Block Diagram of the 2-DOF Robotic Finger.

\section{Results and Discussion}

Fig. 6(a) shows the measured external force $F_{\text {ext }}$ from the embedded force sensor while Fig. 6(b) is the result of modified tipend position of the robotic finger from the proposed positionbased impedance control. The blue line represents the output for $x$ axis direction while red line represents the output for $z$-axis direction. As can be seen in Fig. 6(a), the peaks occurred at time between 10 s and 20 s were resulted when random forces were applied and lifted from the robot's tip-end in both directions. Meanwhile, the result in Fig. 6(b) shows that when the external force in each axis was measured below their $F_{r e f}$, the tip-end position was directed to the targeted position $P_{d}$ which set to [0.04246; $\left.0 ; 0.1245\right]$ However, when the measured external force exceeded their $F_{r e f}$, the $P_{d}$ was modified to $P_{c}$ according to the amount of force applied on the robot's tip-end. Referring to the $x$-axis position-based impedance control output result in Fig. 6(b), when the measured external force exceeded $1 \mathrm{~N}, P_{d}$ was modified to $P_{c}$ as presented by the five peaks. Meanwhile, the z-axis position-based impedance control output result shows that the first measured force applied did not exceed $F_{r e f}$ at $2 \mathrm{~N}$ while other measured force applied exceeded $2 \mathrm{~N}$. From the graph, it can be clearly seen that instead of five, only four forces applied have affected $P_{d}$. The first force applied did not affect $P_{d}$ because it did not exceed $2 \mathrm{~N}$. Conclusively, it can be observed that whenever the force applied exceeded its $F_{r e f}, P_{d}$ will be modified to $P_{c}$ which depends on the amount of applied force to make the robotic finger behaves as a mass-spring-dashpot system.

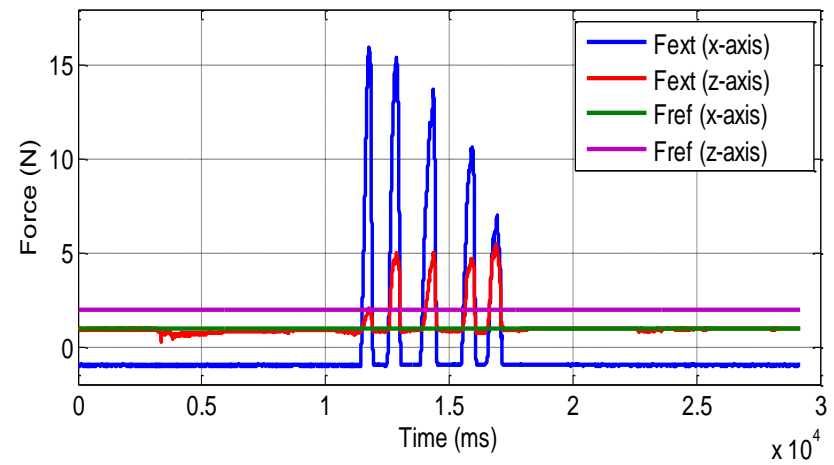

(a) Measured external force $F_{\text {ext }}$. 


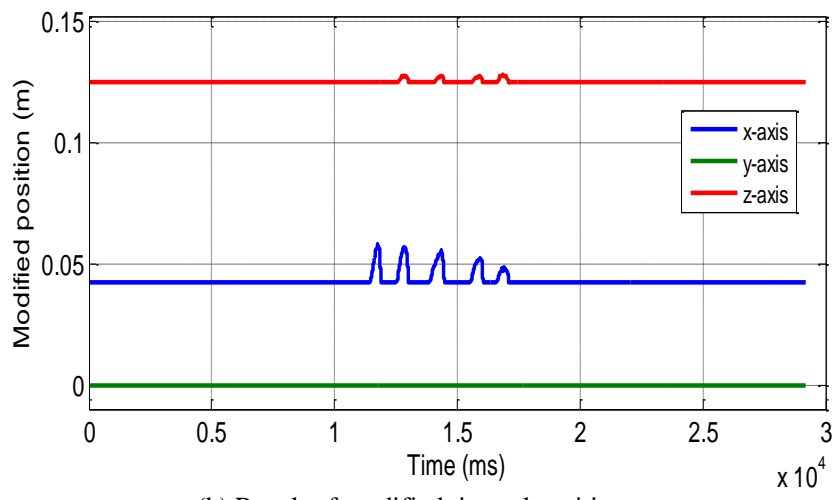

(b) Result of modified tip-end position.

Fig. 6: Result of developed position-based impedance control in $x$-axis and $z$-axis direction.

Next, $P_{c}$ was provided to the derived inverse kinematics to convert the modified tip-end position into angle references to move the joints. The converted angles from $P_{c}$ involved $\theta_{1}$ and $\theta_{2}$ as depicted in Fig. 7. According to the Fig. 7, the tip-end position was initially directed to $25^{\circ}$ and $60^{\circ}$ for $\theta_{1}$ and $\theta_{2}$, respectively. $\theta_{1}$ at $25^{\circ}$ and $\theta_{2}$ at $60^{\circ}$ are the converted angles by the developed inverse kinematics from its targeted position $P_{d}$. Meanwhile, the peaks occurred between time $10 \mathrm{~s}$ and $20 \mathrm{~s}$ in both graphs were the converted angles of modified tip-end position $P_{c}$ which resulted from the activation of position-based impedance control in both directions. During this time, $\theta_{1}$ and $\theta_{2}$ were affected as forces were applied according to Fig. 7 where $\theta_{1}$ increased about $30^{\circ}$ to $37^{\circ}$ from its initial theta at $25^{\circ}$ while $\theta_{2}$ decreased about $49^{\circ}$ to $36^{\circ}$ from its initial theta at $60^{\circ}$. The changes on $\theta_{1}$ and $\theta_{2}$ show that the developed inverse kinematics worked successfully with the position-based impedance control.

$\left({ }^{\circ}\right)$

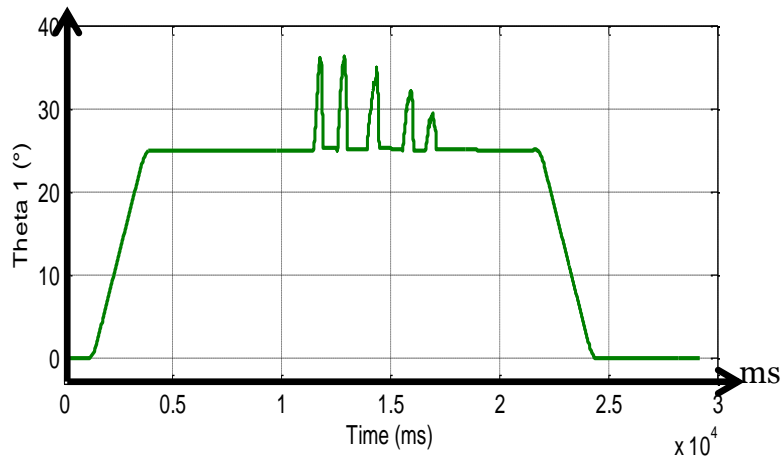

$\left({ }^{\circ}\right)$

(a) Converted angles of $\theta_{1}$

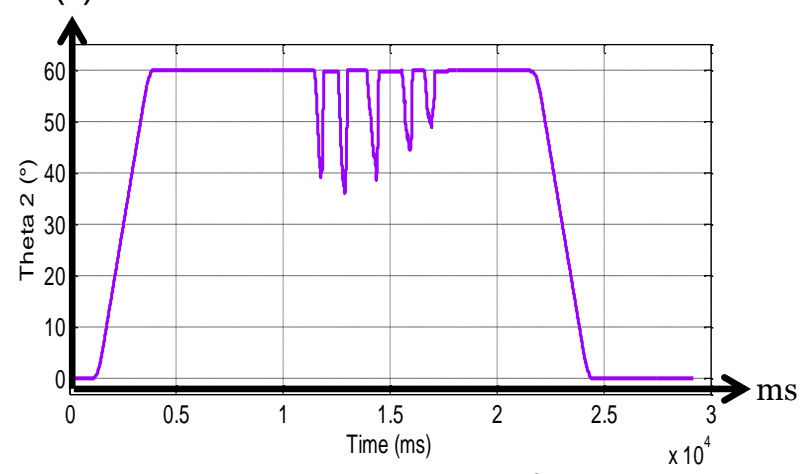

(b) Converted angles of $\theta_{2}$.

Fig. 7: Angles reference converted from tip-end position by the developed inverse kinematics.

Finally, both converted angles were provided as the angle reference to the PID position control to actuate the finger joints by the DC-micromotors for impedance control validation purposes. The result is shown in Fig. 8. According to Fig. 8, the reference angles are the commanded trajectory for motor actuation from the proposed two-axis position-based impedance control.

The commanded trajectory is labeled with green line for $\theta_{1}$ and purple line for $\theta_{2}$. Meanwhile, the actual trajectory of motor actuation resulted from the PID position control is presented in red line for both thetas. From both graphs, it can be observed that the DCmicromotors tend to follow the commanded trajectory from the two-axis position-based impedance control. Small errors between the actual and commanded trajectory with the maximum of $5.5^{\circ}$ and $10.5^{\circ}$ respectively for $\theta_{1}$ and $\theta_{2}$ as shown in Fig. 8 were observed during $11 \mathrm{~s}$ to $17 \mathrm{~s}$. This happened due to the inefficiency of the PID position control to catch up with the fast response of the force control. Even though the DC-micromotors did not exactly move according to the angle references, the actual trajectory is still be able to follow the commanded trajectory. As can be seen in the duration between 10 s and 20s, the 2-DOF robotic finger behaves as a mass-spring-dashpot system through the motion of the DC-micromotors when the tip-end position was modified according to the amount of applied force as shown in Fig. 6(a). Hence the proposed two-axis position-based impedance control is successfully performed the softness to the 2-DOF robotic finger.

$\left({ }^{\circ}\right)$

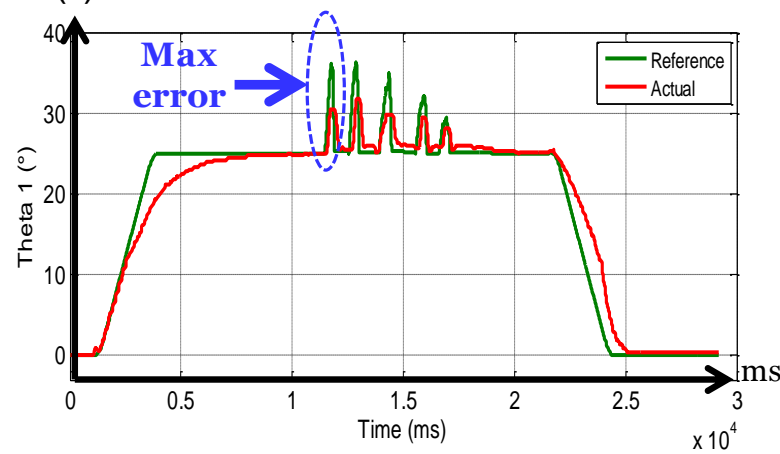

(a) Result of driven motor using PID for $\theta_{1}$.

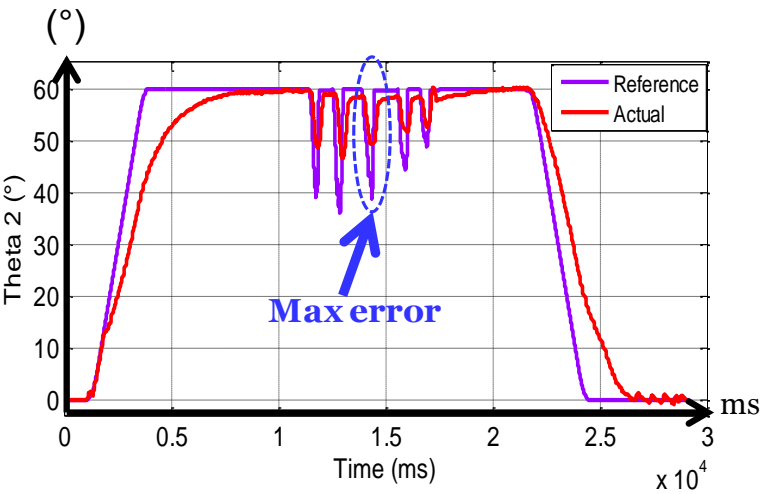

(b) Result of driven motor using PID for $\theta_{2}$

Fig. 8: Real-time experiment result.

\section{Conclusion}

The proposed two-axis position-based impedance control has been developed on one of the 2-DOF finger of the three-fingered robot hand. The experimental result proved that the proposed two-axis position-based impedance control could provide softness to the 2DOF robotic finger by modifying the targeted position $P_{d}$ to a new tip-end position $P_{c}$ when the measured external force $F_{\text {ext }}$ exceeded the force reference $F_{\text {ref }}$. Furthermore, the derived inverse kinematics equation has correctly converted the finger's tipend position in the form of Cartesian position $(\mathrm{X}, \mathrm{Y}, \mathrm{Z})$ to the required position $\left(^{\circ}\right)$ of motor at each joint. Then, once integrated with PID position control, the DC-micromotors was observed to follow the commanded trajectory with maximum position error of $5.5^{\circ}$ and $10.5^{\circ}$, respectively for $\theta_{1}$ and $\theta_{2}$. This was due to the inefficiency of the PID position control to catch up with the fast re- 
sponse of force control will be investigated in the next study. Overall, the effectiveness of the proposed two-axis position-based impedance control and the inverse kinematics solution has been proven successful for the motion of actual robotic finger.

\section{Acknowledgement}

The authors gratefully acknowledge the support from Universiti Teknologi MARA in providing the Academic \& Research Assimilation fund (600-RMI/DANA 5/3/ARAS (11/2015)). The authors would also thank the Faculty of Electrical Engineering, Universiti Teknologi MARA for providing equipments and other supports to conduct this research.

\section{References}

[1] R. V. Patel, H. A. Talebi, J. Jayender, and F. Shadpey, "A Robust Position and Force Control Strategy for 7-DOF Redundan Manipulators," IEEE/ASME Transactions on Mechatronics, vol. 14 pp. 575-589, 2009.

[2] J. G. Garcia, A. Robertsson, J. G. Ortega, and R. Johansson, "Force and Acceleration Sensor Fusion for Compliant Robot Motion Control," in Proc. of the 2005 IEEE Int. Conf. on Robotics and Automation, ICRA, 2005, pp. 2709-2714.

[3] N. Hogan, "Impedance control of industrial robots," Robotics and Computer-Integrated Manufacturing, vol. 1, pp. 97-113, 1984.

[4] H. Z. Arabshahi and A. B. Novinzadeh, "Impedance control of the 3RPS parallel manipulator," in Second RSI/ISM Int. Conf. on Robotics and Mechatronics, ICRoM, 2014, pp. 486-492.

[5] C. Ott, R. Mukherjee, and Y. Nakamura, "Unified Impedance and Admittance Control," in IEEE Int. Conf. on Robotics and Automation, ICRA, 2010, pp. 554-561.

[6] B. Siciliano and L. Villani, Robot force control vol. 540: Springer Science \& Business Media, 2012.

[7] C. Ott, Cartesian impedance control of redundant and flexible-joint robots: Springer, 2008

[8] D. A. Lawrence, "Impedance control stability properties in common implementations," in Proc. IEEE Int. Conf. on Robotics and Automation, 1988, pp. 1185-1190 vol.2.

[9] J. Seul and T. C. Hsia, "Neural network impedance force control of robot manipulator," IEEE Transactions on Industrial Electronics, vol. 45, pp. 451-461, 1998.

[10] M. G. Carmichael and D. Liu, "Admittance control scheme for implementing model-based assistance-as-needed on a robot," in 35th Ann. Int. Conf. of the IEEE Engineering in Medicine and Biology Society, EMBC, 2013, pp. 870-873.

[11] M. K. M. Kasim, R. L. A. Shauri, and K. Nasir, "PID position control of three-fingered hand for different grasping styles," in IEEE 6th Control and System Graduate Research Colloquium, ICSGRC, 2015, pp. 7-10.

[12] J. J. a. R. L. A. Shauri, "Fingertip Structural Analysis-A Simulated Design Evaluation," Jurnal Teknologi, vol. 76, pp. 237-241, 2013.

[13] J. Jaafar, K. Nasir, and R. L. A. Shauri, "Robot Hand Fingertip Design Validation," in 9th International Conference on Robotic, Vision, Signal Processing and Power Applications: Empowering Research and Innovation, H. Ibrahim, S. Iqbal, S. S. Teoh, and M. T. Mustaffa, Eds., ed Singapore: Springer Singapore, 2017, pp. 647-653.

[14] K. Nasir, R. L. A. Shauri, and J. Jaafar, "Calibration of embedded force sensor for robotic hand manipulation," in 2016 IEEE 12th Int Colloquium on Signal Processing \& Its Applications, CSPA, 2016, pp. 351-355.

[15] K. Saiki, "Autonomous assembly work by using arm robot with impedance control," Master's thesis, School of Engineering, Chiba University Graduate School of Engineering, 2012. 